\section{2lcta 3 iologica Sibirica}

Journal of Biology

Founded in 2015
Altai State University

www.asu.ru

ISSN 2412-1908

\title{
New records of long-legged flies (Diptera, Dolichopodidae) from Novosibirsk Region of Russia
}

\author{
I.Ya. Grichanov ${ }^{1}$, O.E. Kosterin ${ }^{2}$, A. Ahmadi ${ }^{3}$ \\ ${ }^{1}$ All-Russian Institute of Plant Protection, Podbelskogo 3, 196608, St.Petersburg-Pushkin, Russia. \\ ${ }^{2}$ Institute of Cytology \& Genetics SB RAS, Acad. Lavrentyev ave. 10, Novosibirsk, 630090, Russia; Novosibirsk \\ State University, Pirogova str. 2, Novosibirsk, 630090, Russia; \\ ${ }^{3}$ Baran Plant Protection Institute, Arak, Markazi Province, Iran \\ E-mail: grichanov@mail.ru; kosterin@bionet.nsc.ru; aezam.ahmadi@gmail.com
}

During a 2017 survey conducted in the Novosibirsk Region of Russia, about 600 specimens of Dolichopodidae were collected. Almost all 44 identified species are recorded for the first time in the Novosibirsk Region.

Key words: Dolichopodidae; Novosibirsk Region; new records

\section{Introduction}

The Novosibirsk Region is one with the poorly studied fauna of long-legged flies in Siberia and Russia (Negrobov et al., 2013; Grichanov, 2017). The first professional contribution to the fauna of Dolichopodidae of the Region was made by O.P. Negrobov who treated material collected by some forestry ecologists. He reported Medetera fasciata Frey, 1915 (Negrobov, 1971a), M. abstrusa Thuneberg, 1955; M. impigra Collin, 1941 (Negrobov, 1971b), M. obscura (Zetterstedt, 1838); M. pseudoapicalis Thuneberg, 1955; M. setiventris Thuneberg, 1955; M. striata Parent, 1927; M. subtristis Negrobov, $1970 ;$ M. thunebergi Negrobov, 1967; and described new species Medetera sibirica Negrobov, 1972, and M. tarasovae Negrobov, 1972, from this Region (Negrobov, Stackelberg, 1971-1977). Subsequently, some of these Medetera species names were repeated in a few ecological reports on entomophages of forest pests (see references in Grichanov \& Negrobov, 1979). In addition to 11 Medetera species, Campsicnemus picticornis (Zetterstedt, 1843), Sciapus albifrons (Meigen, 1830), S. longulus (Fallén, 1823) and Syntormon pumilus (Meigen, 1824) were later found in the Krasnozyorskiy District of the Region (Grichanov, 1998, 2012, 2013). Maslova (2006) recorded Chrysotus viridifemoratus von Roser, 1840, from the Kochkovsky District of the same Region, Negrobov et al. (2010) - Dolichopus latipennis Fallén, 1823, from the environs of Karasuk, Kerchev (2013) - Medetera penicillata Negrobov, 1970, from the Maslyaninskiy District of the Region, and Kornev et al. (2013) recorded Dolichopus simius Parent, 1927, from Novosibirsk.

\section{Material and methods}

In late July/early August 2017, about 600 specimens have been sampled by authors of this paper in the Novosibirsk Region by standard sweep net mostly. Some specimens (e.g., Medetera sp.) have been collected by use of a specimen jar. In addition, two small collections of dolichopodid flies sampled previously were found in the Zoological Institute of the Russian Academy of Sciences, St. Petersburg (ZIN), and the Zoological Museum of Moscow State University, Moscow, Russia (ZMMU). The general species distribution is given after Negrobov et al. (2013) and Grichanov (2017). Type localities are provided, countries are listed as grouped by large regions and then alphabetically. The collectors of all specimens 
Grichanov, I.Ya., et al. (2017). New records of long-legged.... Acta Biologica Sibirica, 2017, 3(4), 20-30

were the authors of this paper unless otherwise is noted. Photos of some habitats for species newly recorded from the Novosibirsk Region are provided. The specimens will be deposited in collections of ZIN and ZMMU.

\section{New records of Dolichopodidae}

\section{Argyra Macquart, 1834 \\ Argyra ilonae Gosseries, 1989}

MATERIAL. Novosibirsk, Akademgorodok env., Botanical Garden and adjacent forest, 30.VII.2017, 1 q.

DISTRIBUTION. Type locality: Denmark. Caucasus, Europe. New for Asia.

REMARKS. The female examined keys to A. ilonae (Grichanov, 2006, 2007), but can represent a different species.

\section{Argyra leucocephala (Meigen, 1824)}

MATERIAL. Novosibirsk Academy Town, Zyryanka rivulet, 14.VI.2008, Kosterin, 1 ऊెं; Novosibirsk, Akademgorodok env., Zyryanka rivulet lower reach, 4.VIII.2017, 2\%; Novosibirsk, Seyatel station env., Shlyuzovskoi Les swampy forest between Pravye Chemy and Nizhnyaya Eltsovka estates, 7.VIII.2017, 1 i ; Novosibirsk, Akademgorodok, Kainka Lake bank, 9.VIII.2017, 4ㅇ.

DISTRIBUTION. Type locality: not given [Europe]. Asia (Iran, Israel, Turkey), Caucasus, Europe, North Africa. The easternmost point of distribution.

\section{Argyra vestita (Wiedemann, 1817)}

MATERIAL. Novosibirsk Akademgorodok, pond in botanical garden, $54.825^{\circ} \mathrm{N}, 83.113^{\circ} \mathrm{E}, 1 . \mathrm{VII} .2009$, Kosterin, $1 \mathrm{\delta}^{\wedge}$.

DISTRIBUTION. Type locality: Germany: "bei Kiel". Asia (Iran, Israel, Turkey), Caucasus, Europe. The easternmost point of distribution.

\section{Campsicnemus Haliday, 1851}

Campsicnemus curvipes (Fallén, 1823)

MATERIAL. Novosibirsk, Akademgorodok env., Botanical Garden and adjacent forest, 30.VII.2017, 201, 19; Novosibirsk, Akademgorodok env., Zyryanka rivulet lower reach, 4.VIII.2017, 1 个; Novosibirsk Reg., Novososedovo env., Yurmanka, Vydrikha River bank, 6.VIII.2017, 5; Novosibirsk, Seyatel station env., Shlyuzovskoi Les swampy forest between Pravye Chemy and Nizhnyaya Eltsovka estates, 7.VIII.2017, $1 \hat{\jmath}$.

DISTRIBUTION. Type locality: not given [Europe]. Asia (Iran, Turkey, Russia: Krasnoyarsk), Caucasus, Europe, North Africa.

\section{Campsicnemus lumbatus Loew, 1857}

MATERIAL. Novosibirsk Reg., Evsino env., Shipunikha River bank, 29.VII.2017, 2o; Novosibirsk Reg., Cherepanovo env., pond at Talmenka River, 29.VII.2017, 30̂, 3\%; Novosibirsk, Akademgorodok env., Botanical Garden and adjacent

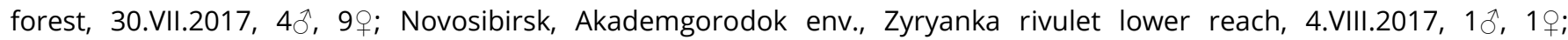
Novosibirsk, Seyatel station env., Shlyuzovskoi Les swampy forest between Pravye Chemy and Nizhnyaya Eltsovka estates, 7.VIII.2017, 20; Novosibirsk, Akademgorodok, Kainka Lake bank, 9.VIII.2017, 18 , 24; Novosibirsk, Inya River lower reach, right bank at Korel-Inya ski pavilion, 10.VIII.2017, 30̂, 2į; Novosibirsk, Akademgorodok, Serebryanoe Lake bank, 11.VIII.2017, 1 \%.

DISTRIBUTION. Type locality: Poland: "aus hiesiger Gegend" [= Meseritz]. Asia (Kazakhstan, Russia: Krasnoyarsk), Caucasus, Europe.

\section{Campsicnemus picticornis (Zetterstedt, 1843)}

REFERENCE. Grichanov, 2012: 250 (Novosibirsk Reg., Krasnozyorskiy Distr.: Polovinnoe village).

MATERIAL. Novosibirsk Reg., Cherepanovo env., pond at Talmenka River, 29.VII.2017, 13i; Novosibirsk, Akademgorodok env., Botanical Garden and adjacent forest, 30.VII.2017, 23̂; Novosibirsk, Akademgorodok, Kainka Lake bank, 9.VIII.2017, 1 ภ, 1 \% .

DISTRIBUTION. Type locality: Sweden: "Suecia meridionali et media, in Scania ad Raften; Ostrogothia ad Larketorp; Haradshammar; Holmiam". Asia (Israel, Kazakhstan, Kyrgyzstan, Turkey, Uzbekistan, Russia: Birobidzhan, Blagoveshchensk, Buryatia, Kamchatka, Khabarovsk, Krasnoyarsk, Novosibirsk, Sayan Mts., Yakutia, Vladivostok), Europe.

Campsicnemus scambus (Fallén, 1823)

MATERIAL. Novosibirsk Akademgorodok, Zyryanka rivulet and pond, pine forest, 3.VIII-2.IX.2007, Kosterin, 13 ; Novosibirsk Academy Town, Zyryanka rivulet, 14.VI.2008, Kosterin, $1{ }^{\lambda}$; Novosibirsk Reg., Evsino env., Shipunikha River bank, 29.VII.2017, 10 ; Novosibirsk, Akademgorodok env., Botanical Garden and adjacent forest, 30.VII.2017, 30ิ, 15; Novosibirsk, Akademgorodok env., Zyryanka rivulet lower reach, 4.VIII.2017, 13; Novosibirsk, Seyatel station env., Shlyuzovskoi Les swampy forest between Pravye Chemy and Nizhnyaya Eltsovka estates, 7.VIII.2017, 24र, 23 q.

DISTRIBUTION. Type locality: Denmark: Rosenthal, Gryphium. Asia (Iran, Mongolia, Turkey, Russia: Altai Rep., Chelyabinsk, Irkutsk, Kamchatka, Khabarovsk, Khantia-Mansia, Krasnoyarsk, Vladivostok, Yamal), Europe, North and Tropical Africa.

\section{Chrysotus Meigen, 1824}

Chrysotus cilipes Meigen, 1824

MATERIAL. Novosibirsk, Akademgorodok env., Ob Reservoir beach and adjacent forest, 31.VII.2017, 3 q.

DISTRIBUTION. Type locality: Germany: Hamburg. Asia (China, Japan, Kazakhstan, Korea, Mongolia, Turkey, Russia: Altai Rep., Baikal, Blagoveshchensk, Chita, Krasnoyarsk, Tomsk, Vladivostok, Yakutia), Caucasus, Europe. 
Grichanov, I.Ya., et al. (2017). New records of long-legged.... Acta Biologica Sibirica, 2017, 3(4), 20-30

\section{Chrysotus laesus (Wiedemann, 1817)}

MATERIAL. Novosibirsk Reg., Krasnozyorskiy Distr.: Polovinnoe village, 21.VI.1988, Knor, Kirov, 1 ภิ.

DISTRIBUTION. Type locality: Germany: Kiel. Asia (China, Kazakhstan, Tajikistan, Turkey, Russia: Altai Rep., Blagoveshchensk, Buryatia, Irkutsk, Krasnoyarsk, Magadan, Orenburg, Omsk, Tomsk, Vladivostok, Yakutia), Caucasus, Europe.

\section{Chrysotus neglectus (Wiedemann, 1817)}

MATERIAL. Novosibirsk, Seyatel station env., Shlyuzovskoi Les swampy forest between Pravye Chemy and Nizhnyaya Eltsovka estates, 7.VIII.2017, 1 \%.

DISTRIBUTION. Type locality: “Holstein”. Asia (Iran, Kazakhstan, Kyrgyzstan, Mongolia, Tajikistan, Uzbekistan, Russia: Altai Rep., Blagoveshchensk, Buryatia, Chelyabinsk, Chita, Kamchatka, Khabarovsk, Krasnoyarsk, Omsk, Tomsk, Vladivostok, Yakutia), Caucasus, Europe.

\section{Chrysotus suavis Loew, 1857}

MATERIAL. Novosibirsk, Akademgorodok, Kainka Lake bank, 9.VIII.2017, 19; Novosibirsk, Inya River lower reach, right bank at Korel-Inya ski pavilion, 10.VIII.2017, 1 ㅇ․

DISTRIBUTION. Type locality: Germany: "Coln"; Austria: "Neusiedler See in Ungarn”. Asia (Afghanistan, Palaearctic China, Iran, Iraq, Israel, Kyrgyzstan, Middle Asia, Mongolia, Turkey, Russia: Altai Rep., Blagoveshchensk, Buryatia, Irkutsk, Kamchatka, Khabarovsk, Krasnoyarsk, Magadan, Sakhalin, Yakutia), Caucasus, Europe, North Africa.

\section{Diaphorus Meigen, 1824}

Diaphorus sp.

REMARKS. Sibling Diaphorus species are hardly distinguished by females; therefore, 5 females collected in various habitats are left unidentified, because they apparently belong to two species different from the following one.

\section{Diaphorus winthemi Meigen, 1824}

MATERIAL. Novosibirsk Reg., Evsino env., Shipunikha River bank, 29.VII.2017, 1 \%; Novosibirsk, Akademgorodok env., Zyryanka rivulet lower reach, 4.VIII.2017, 19; Novosibirsk Reg., Novososedovo env., Yurmanka, Vydrikha River bank, 6.VIII.2017, 1 万, 1 우.

DISTRIBUTION. Type locality: not given. Asia (Turkey, Russia: Krasnoyarsk, Orenburg, Vladivostok), Europe.

Dolichopus Latreille, 1796

\section{Dolichopus agilis Meigen, 1824}

MATERIAL. Novosibirsk Reg., Krasnozyorskiy Distr.: Polovinnoe village, 21.VI.1988, Knor, Kirov, 1 ; ; Novosibirsk Reg., Evsino env., Shipunikha River bank, 29.VII.2017, 1 \%.

DISTRIBUTION. Type locality: not given. Asia (Palaearctic China, Kazakhstan, Mongolia, Russia: Altai Rep., Blagoveshchensk, Buryatia, Irkutsk, Kuril Is., Sayan Mts., Vladivostok, Yakutia), Europe.

\section{Dolichopus argyrotarsis Wahlberg, 1850}

MATERIAL. Novosibirsk Reg., Krasnozyorskoe, 21.VI.1988, Grichanov, 10̄; Novosibirsk Academy Town, Zyryanka rivulet, 14.VI.2008, Kosterin, $7 \overbrace{}^{\top}$ ㅇ.

DISTRIBUTION. Type locality: Sweden: Scania, Provincia Calmariensi. Caucasus, Europe. New for Asia.

\section{Dolichopus campestris Meigen, 1824}

MATERIAL. Novosibirsk Reg., Krasnozyorskoe, 21.VI.1988, Grichanov, $1{ }^{\circledR}$; Novosibirsk Academy Town, 54.8 N, 83.1 E, 18.VI.2009, Kosterin, 13; Novosibirsk Reg., Cherepanovo env., pond at Talmenka River, 29.VII.2017, 1 \%.

DISTRIBUTION. Type locality: not given. Asia (Iran, Kazakhstan, Kyrgyzstan, Turkey, Russia: Altai Rep., Kamchatka, Khabarovsk, Vladivostok), Caucasus, Europe.

\section{Dolichopus clavipes Haliday, 1832}

MATERIAL. Novosibirsk Academy Town, Zyryanka rivulet, 14.VI.2008, Kosterin, $1 \delta^{\Uparrow}$.

DISTRIBUTION. Type locality: Ireland: Holywood. Asia (Palaearctic China, Iran, Kazakhstan, Mongolia, Tajikistan,

Uzbekistan, Russia: Buryatia, Irkutsk, Krasnoyarsk, Orenburg, Yakutia), Caucasus, Europe.

\section{Dolichopus Iatilimbatus Macquart, 1827}

MATERIAL. Novosibirsk, Seyatel station env., Shlyuzovskoi Les swampy forest between Pravye Chemy and Nizhnyaya Eltsovka estates, 7.VIII.2017, 1 ; N Novosibirsk, Akademgorodok, Kainka Lake bank, 9.VIII.2017, $8 \hat{\jmath}$ \% .

DISTRIBUTION. Type locality: not given [“Nord de France"]. Asia (Iran, Kazakhstan, Mongolia, Turkey, Uzbekistan, Russia: Ural, Yakutia), Caucasus, Europe.

\section{Dolichopus linearis Meigen, 1824}

MATERIAL. Novosibirsk Reg., Cherepanovo env., pond at Talmenka River, 29.VII.2017, 2§ơ ; Novosibirsk Reg., Evsino env., Shipunikha River bank, 29.VII.2017, 12 $\hat{\text { }}$; ; Novosibirsk, Akademgorodok env., Botanical Garden and adjacent forest, 30.VII.2017, 19; Novosibirsk, Akademgorodok env., Zyryanka rivulet lower reach, 4.VIII.2017, 19; Novosibirsk Reg., Novososedovo env., Yurmanka, Vydrikha River bank, 6.VIII.2017, 4ð우 Novosibirsk, Inya River lower reach, right bank at Korel-Inya ski pavilion, 10.VIII.2017, 19; Novosibirsk, Akademgorodok, Serebryanoe Lake bank, 11.VIII.2017, 1 ô.

DISTRIBUTION. Type locality: not given. Asia (Palaearctic China, Kazakhstan, Mongolia, Russia: Baikal, KhantiaMansia, Vladivostok), Caucasus, Europe.

Dolichopus longicornis Stannius, 1831 
Grichanov, I.Ya., et al. (2017). New records of long-legged.... Acta Biologica Sibirica, 2017, 3(4), 20-30

MATERIAL. Novosibirsk Reg., Evsino env., Shipunikha River bank, 29.VII.2017, 2q; Novosibirsk, Seyatel station env., Shlyuzovskoi Les swampy forest between Pravye Chemy and Nizhnyaya Eltsovka estates, 7.VIII.2017, 1\%; Novosibirsk, Akademgorodok, Serebryanoe Lake bank, 11.VIII.2017, 301.

DISTRIBUTION. Type locality: not given. Asia (Palaearctic China, Kazakhstan, Mongolia, Russia: Altai Rep., Blagoveshchensk, Irkutsk, Kamchatka, Krasnoyarsk, Magadan, Moscow, Sakhalin, Sayan Mts., Ural, Vladivostok, Yakutia), Caucasus, Europe; Nearctic: Canada (Yukon), USA (Alaska).

Dolichopus longitarsis Stannius, 1831

MATERIAL. Novosibirsk, Akademgorodok env., Botanical Garden and adjacent forest, 30.VII.2017, $2 \hat{\jmath}$..

DISTRIBUTION. Type locality: Germany: Hamburg. Asia (Iran, Kazakhstan, Turkey, Russia: Altai Rep., Krasnoyarsk, Yakutia), Caucasus, Europe.

Dolichopus martynovi Stackelberg, 1930

MATERIAL. Novosibirsk Reg., Cherepanovo env., pond at Talmenka River, 29.VII.2017, 19; Novosibirsk, Seyatel station env., Shlyuzovskoi Les swampy forest between Pravye Chemy and Nizhnyaya Eltsovka estates, 7.VIII.2017, $2 \hat{\jmath}$ o .

DISTRIBUTION. Type locality: Russia: "Siberiae orientalis prov. Austro-Ussuriensis propre pagum Tigrovaja, distr. Sutshanicus; litus meridionalis laci Chanka promotorium Rjabokonj; prope pagum Staraja Devitza, pagum KamenjRybolov; Vladivostok". Asia (Palaearctic China, Kazakhstan, Mongolia, Russia: Irkutsk, Khabarovsk, Vladivostok).

Dolichopus plumipes (Scopoli, 1763)

MATERIAL. Novosibirsk Reg., Cherepanovo env., pond at Talmenka River, 29.VII.2017, 83́f; Novosibirsk, Akademgorodok env., Botanical Garden and adjacent forest, 30.VII.2017, 16 ${ }^{\wedge}$; Novosibirsk, Akademgorodok env., Ob Reservoir beach and adjacent forest, 31.VII.2017, 1+; Novosibirsk Reg., Novososedovo env., Yurmanka, Vydrikha River bank, 6.VIII.2017, 2§ơ; Novosibirsk, Seyatel station env., Shlyuzovskoi Les swampy forest between Pravye Chemy and

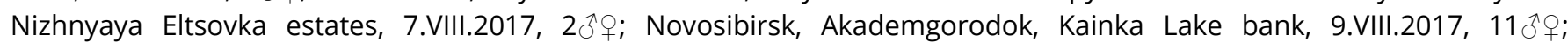

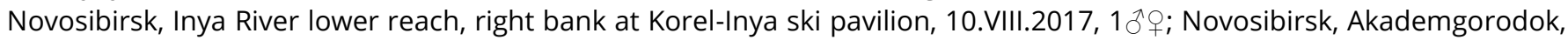
Serebryanoe Lake bank, 11.VIII.2017, 6ð우.

DISTRIBUTION. Type locality: Slovenia: "Carnioliae indigena". Asia (Afghanistan, Palaearctic China, Kazakhstan, Mongolia, Turkey, Uzbekistan, Russia: Baikal, Chukotka, Kamchatka, Khabarovsk, Khantia-Mansia, Koryakia, Tomsk, Tyumen, Vladivostok), Caucasus, Europe; Nearctic, Neotropical and Oriental Regions.

Dolichopus plumitarsis (Fallén, 1823)

MATERIAL. Novosibirsk Reg., Evsino env., Shipunikha River bank, 29.VII.2017, 1 ऊ; Novosibirsk, Akademgorodok env., Ob Reservoir beach and adjacent forest, 31.VII.2017, 1 \% .

DISTRIBUTION. Type locality: Sweden. Asia (Palaearctic China, Kazakhstan, Russia: Altai Rep., Buryatia, Chita, Commander Is., Irkutsk, Khabarovsk, Krasnoyarsk, Kuril Is., Sakhalin, Ural, Vladivostok, Yakutia), Caucasus, Europe; Nearctic: Canada (Ontario), USA (Alaska).

Dolichopus ungulatus (Linnaeus, 1758)

MATERIAL. Novosibirsk, Akademgorodok env., Zyryanka rivulet lower reach, 4.VIII.2017, 10; lower reach, right bank at Korel-Inya ski pavilion, 10.VIII.2017, $2{ }^{\circ}$ \%

DISTRIBUTION. Type locality: Europe. Asia (Palaearctic China, Iran, Kazakhstan, Russia: Altai Rep., Irkutsk, Khabarovsk, Khantia-Mansia, Krasnoyarsk, Orenburg), Caucasus, Europe.

Gymnopternus Loew, 1857

Gymnopternus celer (Meigen, 1824)

MATERIAL. Novosibirsk Academy Town, Zyryanka rivulet, 14.VI.2008, Kosterin, $10^{1}$.

DISTRIBUTION. Type locality: not given. Asia (Kazakhstan, Turkey, Russia: Altai Rep, Buryatia, Krasnoyarsk, Ural), Caucasus, Europe.

Gymnopternus metallicus (Stannius, 1831)

MATERIAL. Novosibirsk, Akademgorodok env., Botanical Garden and adjacent forest, 30.VII.2017, 20ิ, 1; Novosibirsk, Seyatel station env., Shlyuzovskoi Les swampy forest between Pravye Chemy and Nizhnyaya Eltsovka estates, 7.VIII.2017, 1 ․

DISTRIBUTION. Type locality: Germany: “Umgegend von Hamburg”. Asia (Iran, Kazakhstan, Turkey, Russia: Altai Rep., Kamchatka, Khabarovsk, Krasnoyarsk, Vladivostok), Caucasus, Europe.

Hercostomus Loew, 1857

Hercostomus fulvicaudis (Haliday, 1851)

MATERIAL. Novosibirsk, Akademgorodok env., Zyryanka rivulet lower reach, 4.VIII.2017, 1 9.

DISTRIBUTION. Type locality: England. Asia (Palaearctic China, Iran, Kazakhstan, Tajikistan, Turkey, Turkmenistan),

Europe. New for Asian part of Russia.

Hercostomus nigriplantis (Stannius, 1831)

MATERIAL. Novosibirsk, Akademgorodok env., Ob Reservoir beach and adjacent forest, 31.VII.2017, 1 q.

DISTRIBUTION. Type locality: Germany: Potsdam, Berlin. Asia (Russia: Buryatia), Caucasus, Europe.

Hercostomus rusticus (Meigen, 1824)

MATERIAL. Novosibirsk reg, Salairskii env., Suenga River valley, 5434'30"N, 8451'12"E, 6.VII.2008, Kosterin, $10^{\wedge}$.

DISTRIBUTION. Type locality: not given. Asia (Iran, Kazakhstan, Kyrgyzstan, Mongolia, Russia: Altai Rep., Blagoveshchensk, Buryatia, Chita, Irkutsk, Krasnoyarsk, Omsk, Ural, Vladivostok, Yakutia), Caucasus, Europe. 
Grichanov, I.Ya., et al. (2017). New records of long-legged.... Acta Biologica Sibirica, 2017, 3(4), 20-30

\section{Hercostomus udeorum Stackelberg, 1933}

MATERIAL. Novosibirsk, Inya River lower reach, right bank at Korel-Inya ski pavilion, 10.VIII.2017, 1 q.

DISTRIBUTION. Type locality: Russia: “Ussuri-Gebiet bei der Station Tigrovaya, Distr. Sutshan”. Russia: Altai Rep., Blagoveshchensk, Khabarovsk, Vladivostok.

Hydrophorus Fallén, 1823

Hydrophorus brunnicosus Loew, 1857

MATERIAL. Novosibirsk, Akademgorodok env., Botanical Garden and adjacent forest, 30.VII.2017, 2o; Novosibirsk

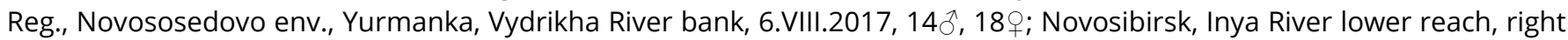
bank at Korel-Inya ski pavilion, 10.VIII.2017, 12ð̂, 5ㅇ.

DISTRIBUTION. Type locality: Poland: Poznan. Asia (Russia: Orenburg, NW Siberia, Krasnoyarsk, Yakutia), Europe.

Hydrophorus callostomus Loew, 1857

MATERIAL. Novosibirsk, Akademgorodok env., Botanical Garden and adjacent forest, 30.VII.2017, 1 9 ; Novosibirsk, Akademgorodok env., Zyryanka rivulet lower reach, 4.VIII.2017, 1q; Novosibirsk, Akademgorodok, Kainka Lake bank, 9.VIII.2017, 1 \%.

DISTRIBUTION. Type locality: Russia: Siberia. Asia (“Middle Asia”, Turkey, Russia: Omsk, Transbaikalia, Ural, Yakutia), Caucasus, Europe.

Lamprochromus Mik, 1878

Lamprochromus semiflavus (Strobl, 1880)

MATERIAL. Novosibirsk, Akademgorodok, Kainka Lake bank, 9.VIII.2017, 1 \%.

DISTRIBUTION. Distribution. Type localitty: Austria: Conventgarten. Asia (Turkey), Europe. The easternmost point of distribution.

Medetera Fischer von Waldheim, 1819

Medetera jacula (Fallén, 1823)

MATERIAL. Novosibirsk, Akademgorodok env., Utinyy Pond, 30.VII.2017, 1+; Novosibirsk, Akademgorodok, Utinyy Pond, on birch tree trunks, 5.VIII.2017, 2ᄎ, 2ᄋ; Novosibirsk Reg., Novososedovo env., Yurmanka, Vydrikha River bank, 6.VIII.2017, 6+; Novosibirsk, Seyatel station env., Shlyuzovskoi Les swampy forest between Pravye Chemy and Nizhnyaya Eltsovka estates, 7.VIII.2017, 3^,19.

DISTRIBUTION. Type locality: Sweden: Scania. Asia (Iran, Kazakhstan, Turkey, Russia: Altai Rep., Buryatia, Irkutsk, Sayan Mts., Urals, Yakutia), Caucasus, Europe, North Africa.

\section{Rhaphium Meigen, 1803}

Rhaphium sp.

REMARKS. Sibling Rhaphium species are hardly distinguished by females; therefore, 31 females collected in various localities are left unidentified, because they apparently belong to more than three species listed below.

\section{Rhaphium caliginosum Meigen, 1824}

MATERIAL. Novosibirsk Reg., Evsino env., Shipunikha River bank, 29.VII.2017, 1§; Novosibirsk, Akademgorodok env., Botanical Garden and adjacent forest, 30.VII.2017, 13ึ; Novosibirsk, Seyatel station env., Shlyuzovskoi Les swampy forest between Pravye Chemy and Nizhnyaya Eltsovka estates, 7.VIII.2017, 10; Novosibirsk, Inya River lower reach, right bank at

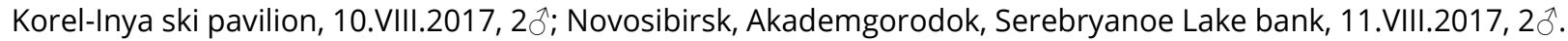

DISTRIBUTION. Type locality: not given. Asia (Golan Heights, Israel, Syria, Turkey, Russia: Krasnoyarsk), Caucasus, Europe, North Africa.

\section{Rhaphium elegantulum (Meigen, 1824)}

MATERIAL. Novosibirsk, Akademgorodok, Kainka Lake bank, 9.VIII.2017, 13 , 2 \%.

DISTRIBUTION. Type locality: Germany: Hamburg. Asia ("Polar Ural”, “Igarka”, Baikal, Kamchatka, Khantia-Mansia, Krasnoyarsk), Caucasus, Europe; Nearctic: USA and Canada.

\section{Rhaphium laticorne (Fallén, 1823)}

MATERIAL. Novosibirsk Reg., Evsino env., Shipunikha River bank, 29.VII.2017, 2§̊; Novosibirsk, Akademgorodok env., Botanical Garden and adjacent forest, 30.VII.2017, 10.

DISTRIBUTION. Type locality: Sweden. Asia (“Middle Asia”, Turkey, Russia: Altai Rep., Krasnoyarsk), Caucasus, Europe.

Sympycnus Loew, 1857

Sympycnus pulicarius (Fallén, 1823)

MATERIAL. Novosibirsk Reg., Evsino env., Shipunikha River bank, 29.VII.2017, 1 \%; Novosibirsk, Akademgorodok env., Botanical Garden and adjacent forest, 30.VII.2017, 1 ; Novosibirsk Reg., Novososedovo env., Yurmanka, Vydrikha River bank, 6.VIII.2017, 3̧̂, 2+; Novosibirsk, Seyatel station env., Shlyuzovskoi Les swampy forest between Pravye Chemy and Nizhnyaya Eltsovka estates, 7.VIII.2017, 1 đ̊; Novosibirsk, Akademgorodok, Kainka Lake bank, 9.VIII.2017, 2+; Novosibirsk, Akademgorodok, Serebryanoe Lake bank, 11.VIII.2017, 2ㅇ.

DISTRIBUTION. Type locality: not given [Sweden]. Asia (Iran, Kazakhstan, Turkey, Russia: Altai Rep., Altai Ter., Buryatia, Chelyabinsk, Khakassia, Krasnoyarsk, Yakutia), Caucasus, Europe; Nearctic Region.

Syntormon Loew, 1857

Syntormon freymuthae Loew, 1873

MATERIAL. Novosibirsk, Akademgorodok, Serebryanoe Lake bank, 11.VIII.2017, 1 ․ 
Grichanov, I.Ya., et al. (2017). New records of long-legged.... Acta Biologica Sibirica, 2017, 3(4), 20-30

DISTRIBUTION. Type locality: Russia: “Nischegrod'schen Gouvernement; bei Malaja Arja in Moskauer Gouvernement". Estonia, Russia (Leningrad, Moscow, Nizhnii Novgorod), "Middle Asia". The easternmost point of distribution.

Teuchophorus Loew, 1857

Teuchophorus sp.

REMARKS. Palaearctic Teuchophorus species are indistinguishable by females; therefore, 23 females collected in various localities are left unidentified, because most localities contain more than one species listed below.

Teuchophorus nigricosta (von Roser, 1840)

MATERIAL. Novosibirsk Reg., Novososedovo env., Yurmanka, Vydrikha River bank, 6.VIII.2017, 20;; Novosibirsk, Seyatel station env., Shlyuzovskoi Les swampy forest between Pravye Chemy and Nizhnyaya Eltsovka estates, 7.VIII.2017, 1§i; Novosibirsk, Akademgorodok, Serebryanoe Lake bank, 11.VIII.2017, $1 \hat{\jmath}$.

DISTRIBUTION. Type locality: not given (Germany: Wurttemberg). Asia (Russia: Altai Ter., Krasnoyarsk), Europe.

\section{Teuchophorus calcaratus (Macquart, 1827)}

MATERIAL. Novosibirsk Reg., Evsino env., Shipunikha River bank, 29.VII.2017, 1§; Novosibirsk, Akademgorodok env., Botanical Garden and adjacent forest, 30.VII.2017, 23; Novosibirsk, Akademgorodok env., Zyryanka rivulet lower reach,

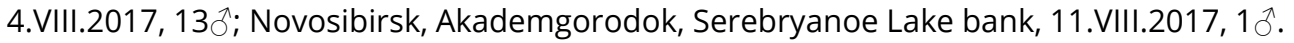

DISTRIBUTION. Type locality: not given (France). Asia (Russia: Krasnoyarsk), Caucasus, Europe.

Teuchophorus spinigerellus (Zetterstedt, 1843)

MATERIAL. Novosibirsk, Seyatel station env., Shlyuzovskoi Les swampy forest between Pravye Chemy and Nizhnyaya Eltsovka estates, 7.VIII.2017, 11 $\delta^{\lambda}$.

DISTRIBUTION. Type locality: "Suecia meridionali et media, Scania ad Lund; Ostrogothia ad Wadstena, Dania" [Sweden, Denmark]. Asia (Kazakhstan, Turkey), Caucasus, Europe. The easternmost point of distribution.

\section{Thinophilus Wahlberg, 1844}

\section{Thinophilus ruficornis (Haliday, 1838)}

MATERIAL. Novosibirsk, Akademgorodok env., Botanical Garden and adjacent forest, 30.VII.2017, 1 ; ; Novosibirsk, Inya River lower reach, right bank at Korel-Inya ski pavilion, 10.VIII.2017, 1 .

DISTRIBUTION. Type locality: England: Tarbert. Asia (Palaearctic China, Kazakhstan, Kyrgyzstan, Mongolia, Russia: Orenburg, Omsk, Yakutia), Caucasus, Europe.

\section{Thrypticus Gerstaecker, 1864}

Thrypticus bellus Loew, 1869

MATERIAL. Novosibirsk Reg., Cherepanovo env., pond at Talmenka River, 29.VII.2017, 19; Novosibirsk, Akademgorodok env., Botanical Garden and adjacent forest, 30.VII.2017, 1 ô.

DISTRIBUTION. Type locality: England: Kew. Asia (Palaearctic China, Golan Heights, Iran, Israel, Kazakhstan, Turkey, Russia: Krasnoyarsk, Vladivostok), Caucasus, Europe, North Africa; Afrotropical Region.

\section{Conclusion}

O.P. Negrobov described two new Medetera species (M. sibirica and M. tarasovae), from the Toguchinskiy District of the Novosibirsk Region (Negrobov, Stackelberg, 1971-1977). Later, a lot of more new species were described from the Palaearctic Region including its eastern part (see review of Negrobov \& Naglis (2016). Therefore, the 11 Medetera species names published by Negrobov $(1971 a, b)$ and repeated in a few ecological reports must be confirmed. Negrobov et al. (2013) mentioned 8 Medetera species for the Novosibirsk Region. In 1988, I. Grichanov participated in an expedition to the Krasnozyorskiy District situated in the South-West of the Region within the rather arid steppe/forest-steppe ecoregion. Eight dolichopodid species were found, including 5 species not recorded elsewhere in the Region (Grichanov, 1998, 2012, 2013, and this paper). O. Kosterin collected several species in 2007-2009 in environs of the Akademgorodok, which were mainly sampled again during our recent survey (see above).

As a result of 2017 survey conducted in the Novosibirsk Region (environs of Novosibirsk; Cherepanovskiy, Iskitimskiy and Maslyaninskiy districts of the Region), a new material of Dolichopodidae was collected and identified. Two old collections of dolichopodid flies were also studied. In all, 44 species were identified, including only one species previously known from the Region. Almost all species were collected along banks of rivers, lakes and other water sources (see Figs. 1-8); nevertheless, Medetera jacula inhabited tree trunks and wooden poles.

Most of collections were made in the forest-steppe zone characterized by alternating meadows and meadow steppes and birch groves. At the same time, many species were found at the Akademgorodok standing in pine forests growing along the Ob River. Some specimens were collected at the foothills of Salairskiy Kryazh in the east of Novosibirsk Region, with mesic pine (at Yurmanka) or fir (at the Suenga River) forests; and some ones in the Krasnozyorskiy District lying in a transition zone from the southern forest-steppe to steppe. According to sampling data, five species (Campsicnemus lumbatus, C. scambus, Dolichopus linearis, D. plumipes and Hydrophorus brunnicosus) had the highest total abundance in 2017.

Most collected species are widespread across the Palaearctic Region. At present, the Novosibirsk Region is the easternmost area of distribution for the following species: Argyra ilonae, A. leucocephala, A. vestita, Dolichopus argyrotarsis, Lamprochromus semiflavus, Sciapus albifrons, S. Iongulus, Syntormon freymuthae, and Teuchophorus spinigerellus. The following species are not known in Europe: Dolichopus martynovi, Hercostomus udeorum, Medetera penicillata, M. sibirica, M. 
subtristis and $M$. tarasovae. As a result of our investigation, the number of reported dolichopodid species from the Region includes now 61 species. We suggest that many more species will be revealed in this Region, if new districts and localities are investigated with the use of mass trapping methods. The total number of species can reach here to 200 dolichopodids.

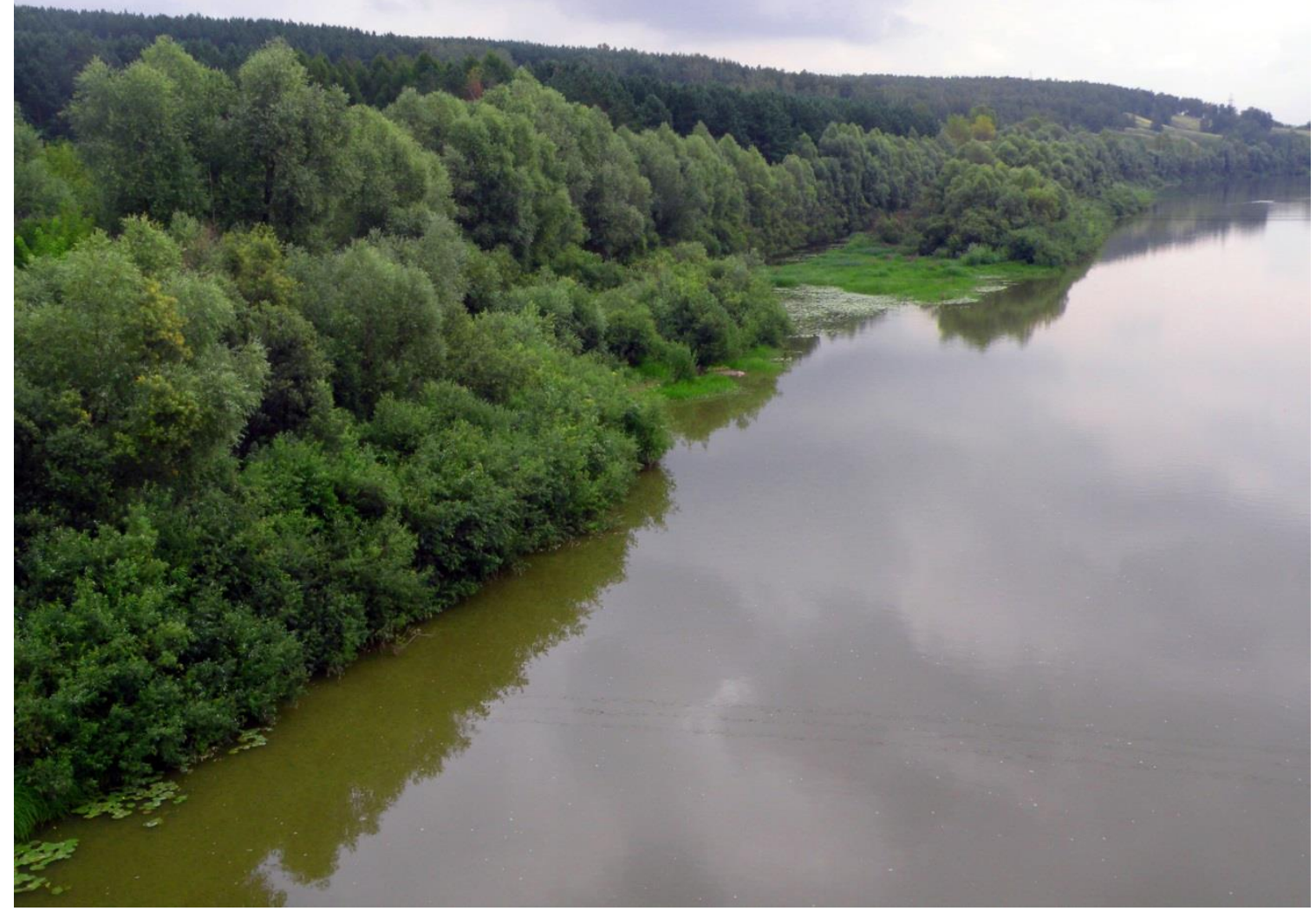

Fig. 1. Inya River lower reach. Novosibirsk, 10 August 2017

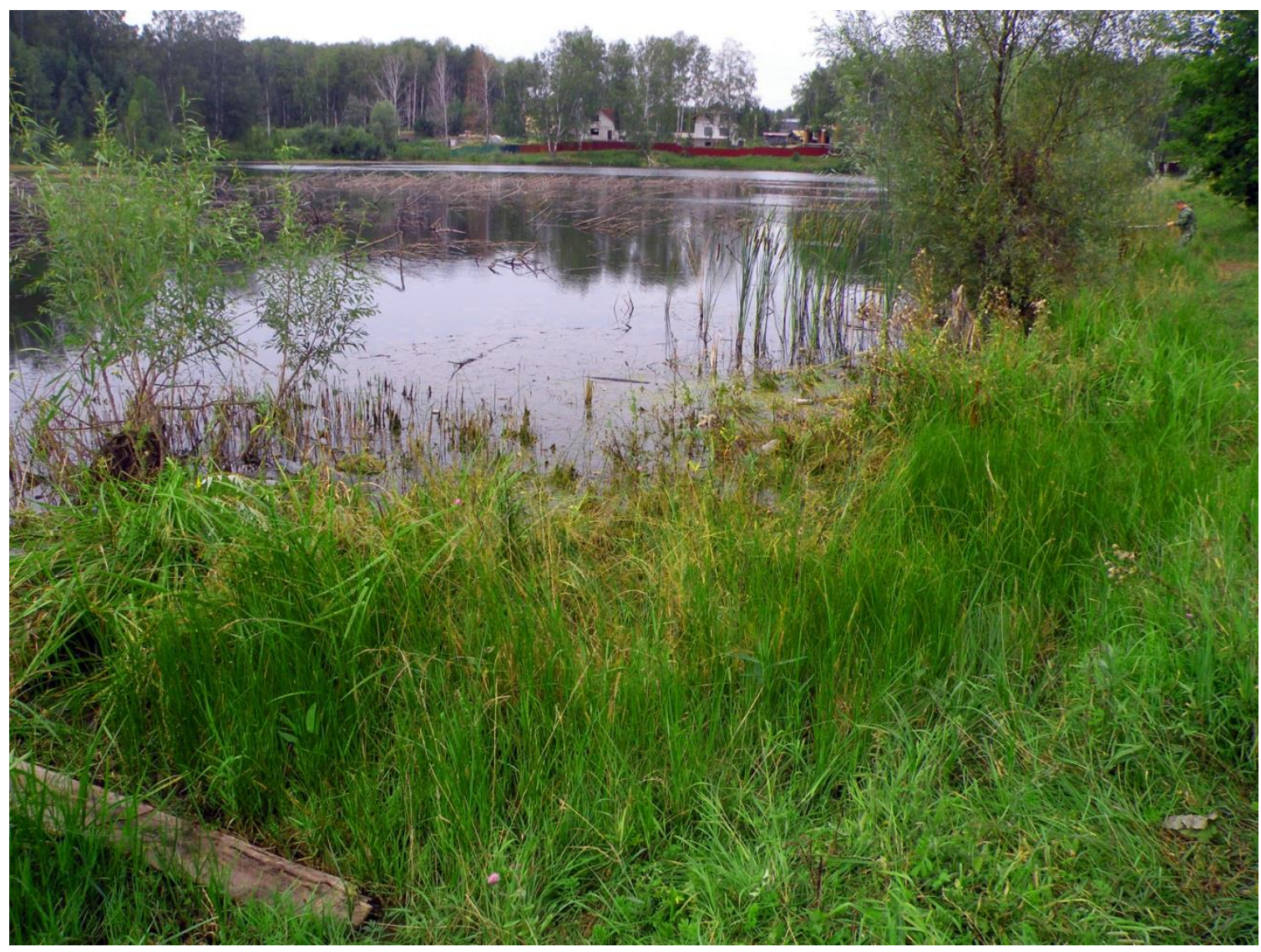

Fig. 2. Kainka Lake bank. Novosibirsk, Akademgorodok, 9 August 2017 


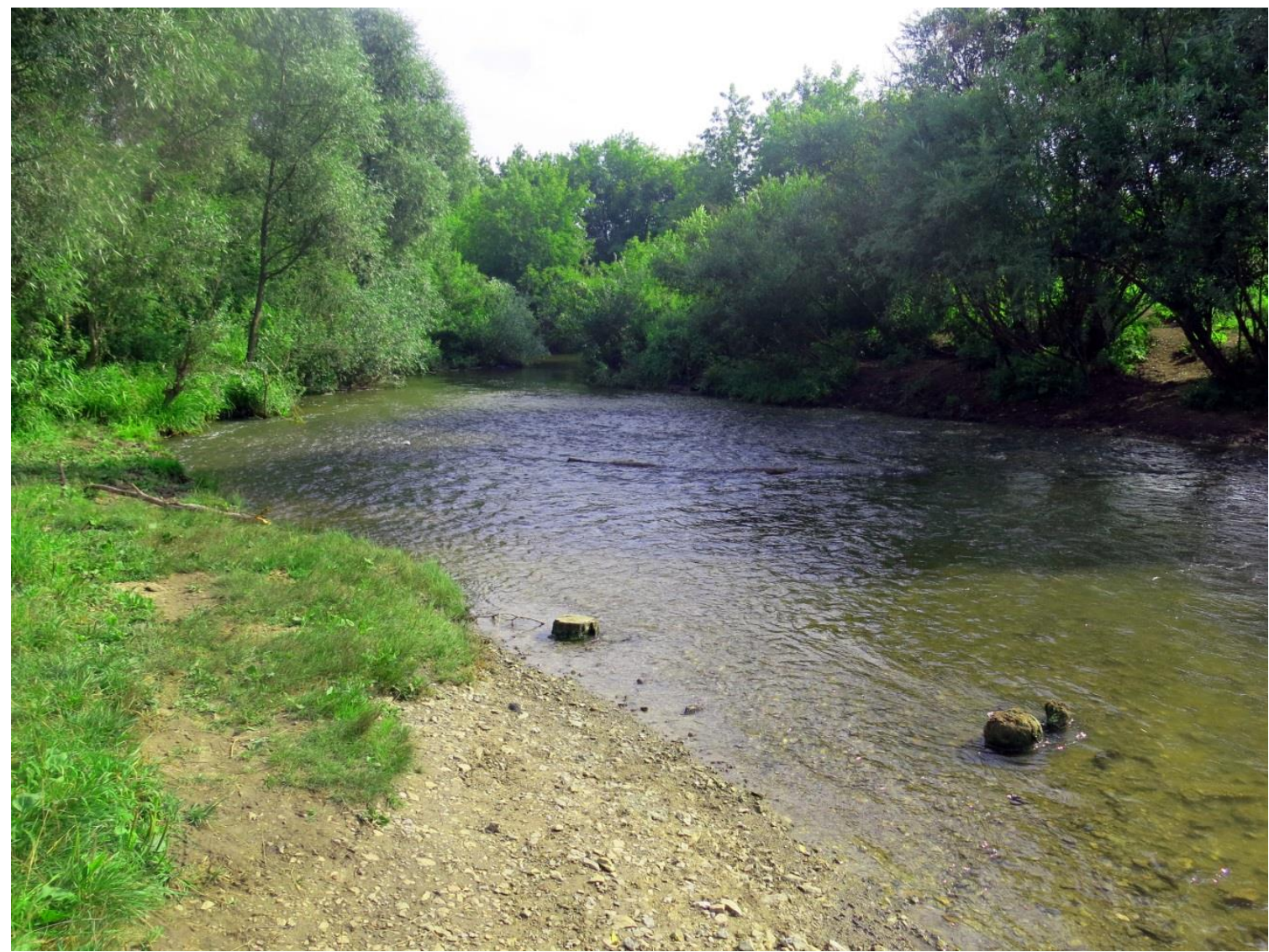

Fig. 3. Shipunikha River. Evsino environs, 29 July 2017

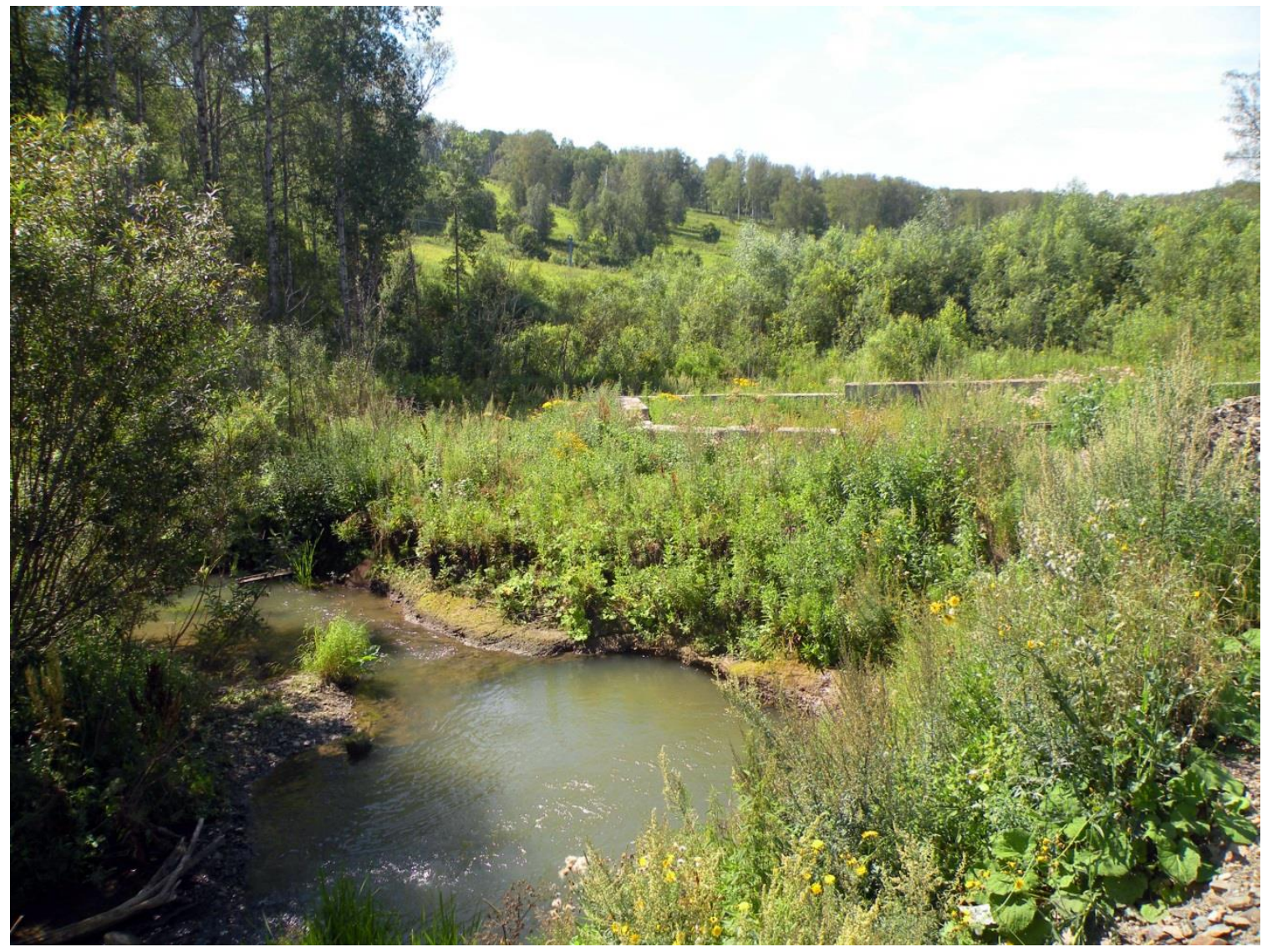

Fig. 4. Vydrikha River. Yurmanka, 6 August 2017 


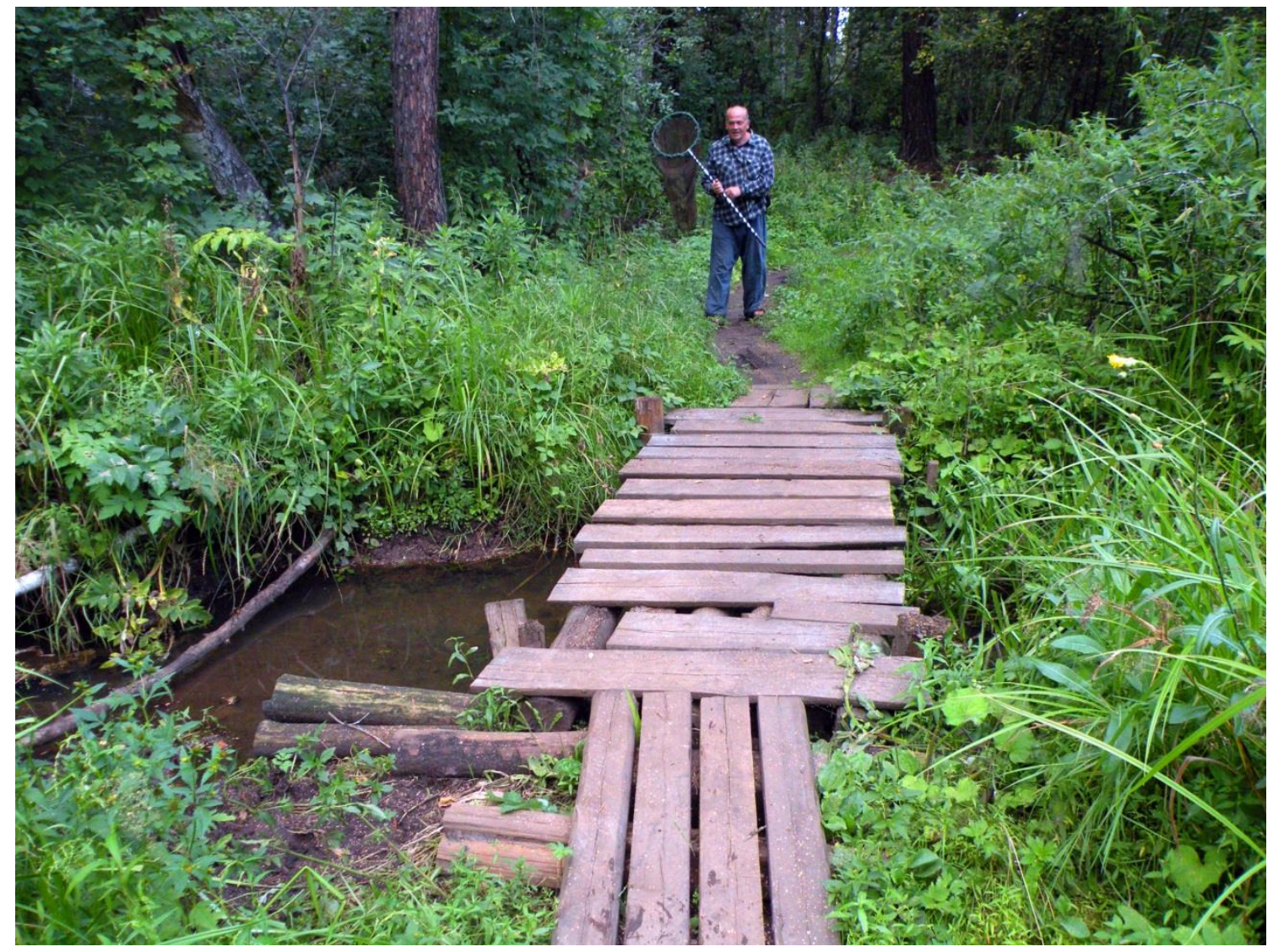

Fig. 5. Shlyuzovskoi Les swampy forest between Pravye Chemy and Nizhnyaya Eltsovka estates. Novosibirsk, Ob River bank, 7 August 2017

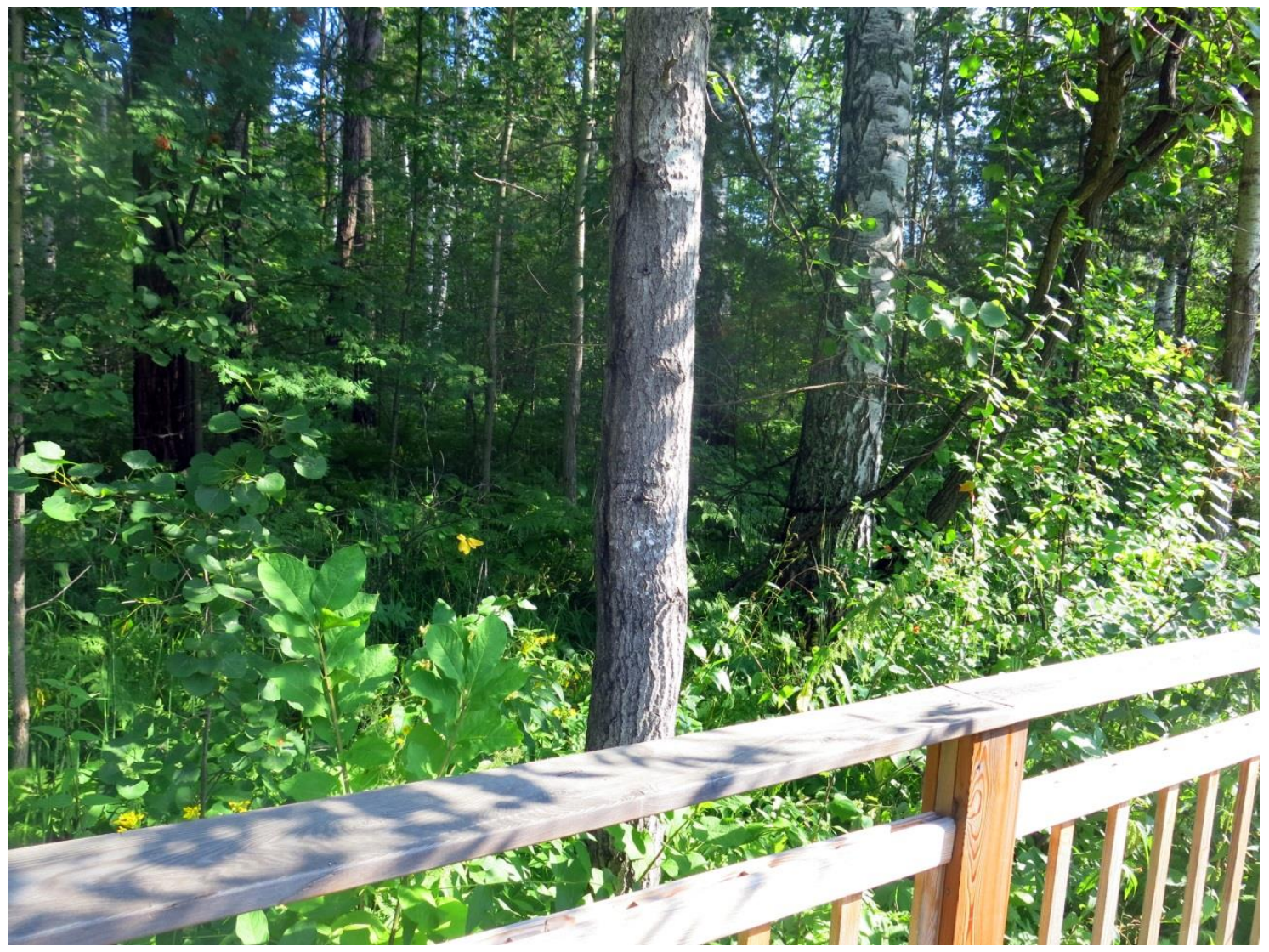

Fig. 6. Birch grove at Utinyy Pond. Novosibirsk, Akademgorodok, 5 August 2017 


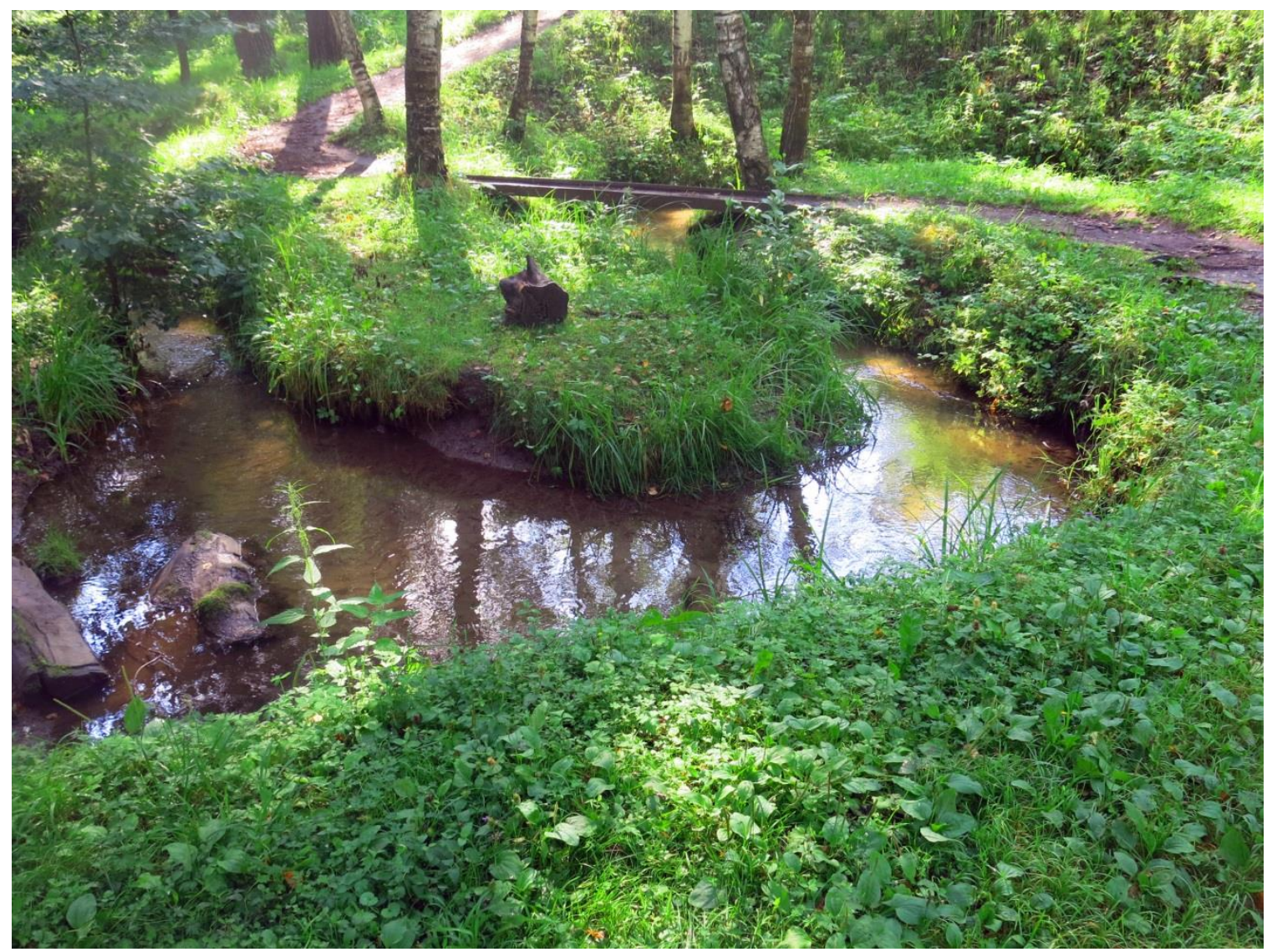

Fig. 7. Zyryanka rivulet in Botanical Garden. Novosibirsk, Akademgorodok, 30 July 2017

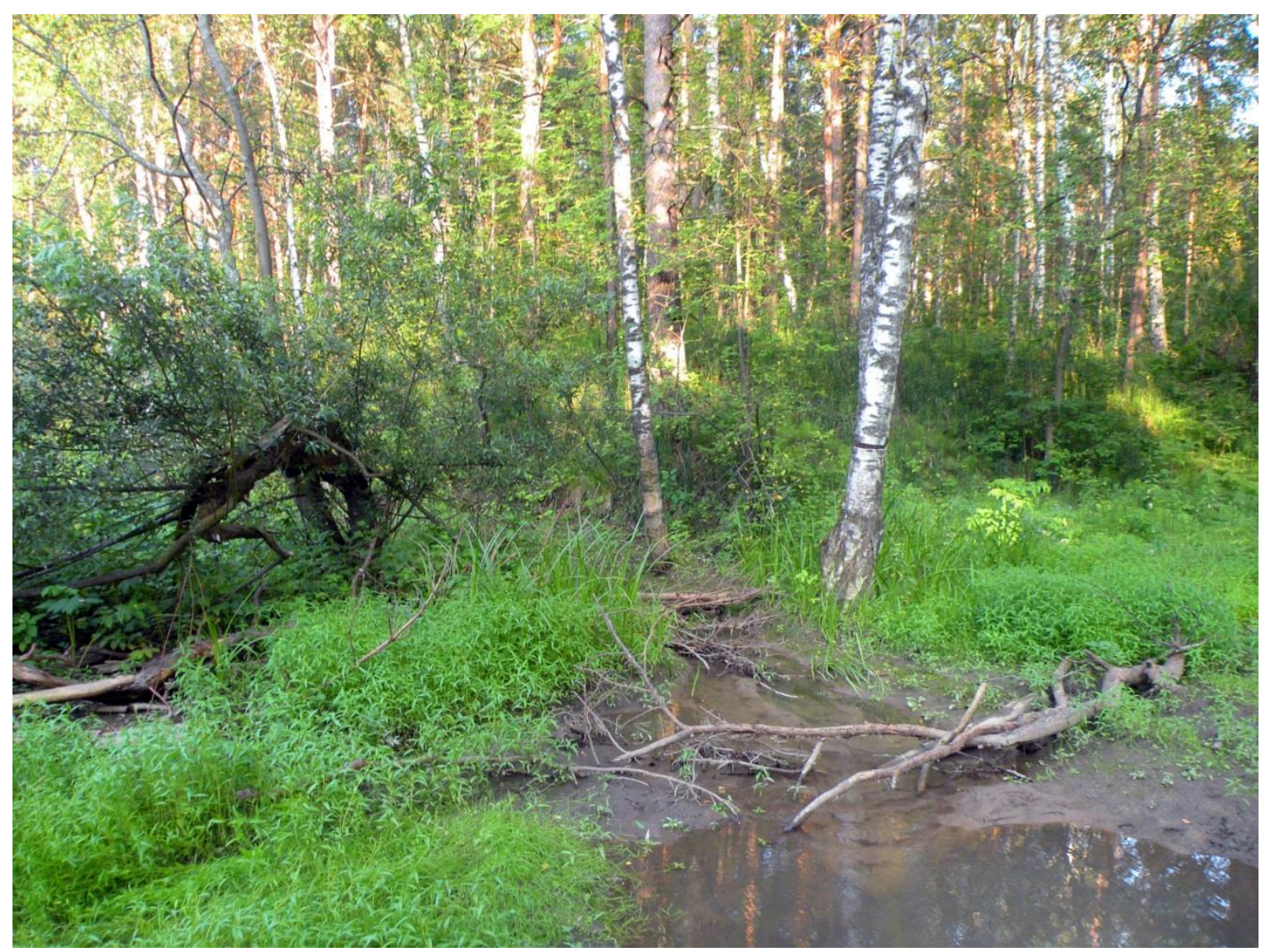

Fig. 8. Zyryanka rivulet lower reach. Novosibirsk, Akademgorodok, 5 August 2017 
Grichanov, I.Ya., et al. (2017). New records of long-legged.... Acta Biologica Sibirica, 2017, 3(4), 20-30

\section{Acknowledgements}

The authors are sincerely grateful to Dr. E.P. Nartshuk and L.A. Kuznetsova (St. Petersburg, Russia), Drs. Andrei Ozerov and Nikita Vikhrev (Moscow, Russia) for their kindness in providing specimens for this study, to Tatyana Kolesnikova for the Evsino-Cherepanovo field trip, and to organizers of the $15^{\text {th }}$ Congress of the Russian Entomological Society for the field trip to Yurmanka.

\section{References}

Grichanov, I.Ya. (1998). New data on distribution of Dolichopodidae (Diptera) species in Russia, Ukraine and Kazakhstan. In: Medvelev, G.S. (Ed.). Problems of Entomology in Russia, vol. 1, 102-104. St. Petersburg: Zoologicheskii Institut RAN (in Russian).

Grichanov, I.Ya. (2006). A checklist and keys to North European genera and species of Dolichopodidae (Diptera). St.Petersburg: VIZR, 1-120 (Plant Protection News Supplements). Available from: https://diptera.info/downloads/Grichanov_N_Europe.pdf (accessed 30 August 2017).

Grichanov, I.Ya. (2007). A checklist and keys to Dolichopodidae (Diptera) of the Caucasus and East Mediterranean. St.Petersburg: VIZR, 1-160 (Plant Protection News Supplements). Available from: http://www.diptera.info/downloads/Grichanov 2007b.pdf (accessed 30 August 2017).

Grichanov, I.Ya. (2012). A new species of Campsicnemus from the Far East of Russia with some new records (Dolichopodidae, Diptera). Amurian zoological journal, 4(3), 250-252.

Grichanov, I.Ya. (2013). Systematic notes on West-Palearctic species of the genus Syntormon Loew (Diptera: Dolichopodidae). In: Grichanov, I.Ya., Negrobov, O.P. (Eds.). Fauna and taxonomy of Dolichopodidae (Diptera). Collection of papers. St.Petersburg: VIZR, 3-26 (Plant Protection News Supplements). Available from https://archive.org/details/GrichanovNegrobovDolichopodidae2013 (accessed 30 August 2017).

Grichanov, I.Ya. (2017). Alphabetic list of generic and specific names of predatory flies of the epifamily Dolichopodoidae (Diptera). 2nd Edition. St.Petersburg: VIZR, 1-563. (Plant Protection News Supplements, N23). Available from: https://archive.org/download/Grichanov2017DolibankSec/Grichanov\%202017\%20Dolibank-sec.pdf (accessed 30 August 2017).

Grichanov, I.Ya., Negrobov, O.P. (1979). Catalogue for the family Dolichopodidae (Diptera) in the USSR. Voronezh: Voronezh University, 1-128 (Dep. in VINITI, Moscow 04.02.80, N417-80 dep.).

Kerchev, I.A. (2013). The interfaced invasion of Polygraphus proximus Blandf. (Coleoptera: Curculionidae, Scolytinae) and its predator Medetera penicillata Neg. (Diptera: Dolichopodidae) to dark-coniferous forests of Siberia. In: Selikhovkin, A.V., Musolin, D.L. (Eds.). The Kataev Memorial Readings - VII. Pests and Diseases of Woody Plants in Russia. Proceedings of the International Conference. Saint Petersburg (Russia), November, 25-27, 2013. St.Petersburg: State Forest Technical University, 44 (in Russian). Available online: http://ftacademy.ru/science/kataev/7threading.

Kornev, I.I., Negrobov, O.P., Selivanova, O.V. (2013). New data on fauna and systematics of Dolichopus simius Parent, 1927 (Dolichopodidae, Diptera). Amurian zoological journal, 5(2), 147-150 (in Russian).

Maslova, O.O. (2006). Revision of the genus Chrysotus (Dolichopodidae, Diptera) of Russia and adjacent territories. Abstract of PhD Thesis (MS). Voronezh: Voronezh LTA, 1-20 (in Russian).

Negrobov, O.P. (1971). Species of the genus Medetera (Dolichopodidae, Diptera) as entomophages of bark beetles. In: Vorontsov, A.I. (Ed.). Zashchita lesa ot vrednykh nasekomykh i boleznei: Tez. dokl. Vsesoyuz. nauch.-tekhn. konf., vol. 3, 89-90. Moscow: MLTI (in Russian).

Negrobov, O.P., Barkalov, A.V., Selivanova, O.V. (2010). New data on the fauna of the family Dolichopodidae (Diptera) from Russia, with a description of a new species of the genus Argyra Mcq. Euroasian Entomological Journal, 9(3), 522-524.

Negrobov, O.P., Naglis, S. (2016). Palaearctic species of the genus Medetera (Diptera: Dolichopodidae). Zoosystematica Rossica, 25(2), 333-379.

Negrobov, O.P., Selivanova, O.V., Maslova, O.O., Chursina M.A. (2013). Check-list of predatory flies of the family Dolichopodidae (Diptera) in the fauna of Russia. In: Grichanov, I.Ya., Negrobov, O.P. (Eds.). Fauna and taxonomy of Dolichopodidae (Diptera). Collection of papers. St.Petersburg: VIZR RAAS (Plant Protection News Suppl.), 47-93. Available from: https://archive.org/details/GrichanovNegrobovDolichopodidae2013 (accessed 30 August 2017).

Negrobov, O.P., Stackelberg, A.A. (1971-1977). Dolichopodidae, Unterfamilie Medeterinae. In: Lindner, E. (Ed.). Die Fliegen der Palaearktischen Region. Stuttgart, IV, 29, 284 (1971), 289 (1972), 302, 303 (1974), 316 (1977), $238-354$.

\section{Citation:}

Grichanov, I.Ya., Kosterin, O.E., Ahmadi, A. (2017). Species composition of Ground beetles (Coleoptera, Carabidae) in Saransk. Acta Biologica Sibirica, 3 (4), 20-30.

Submitted: 08.06.2016. Accepted: 04.08.2017

cross ref http://dx.doi.org/10.14258/abs.v3i4.3630

(C) 2017 by the authors. Submitted for possible open access publication under the terms and conditions of the Creative Commons Attribution (CC BY) license (http://creativecommons.org/licenses/by/4.0/). 